\title{
Passively Controllable Smart Antennas
}

\author{
Javad Lavaei, Aydin Babakhani, Ali Hajimiri and John C. Doyle
}

\begin{abstract}
We recently introduced passively controllable smart (PCS) antenna systems for efficient wireless transmission, with direct applications in wireless sensor networks. A PCS antenna system is accompanied by a tunable passive controller whose adjustment at every signal transmission generates a specific radiation pattern. To reduce co-channel interference and optimize the transmitted power, this antenna can be programmed to transmit data in a desired direction in such a way that no signal is transmitted (to the far field) at pre-specified undesired directions. The controller of a PCS antenna was assumed to be centralized in our previous work, which was an impediment to its implementation. In this work, we study the design of PCS antenna systems under decentralized controllers, which are both practically implementable and cost efficient. The PCS antenna proposed here is made of one active element and its programming needs solving second-order-cone optimizations. These properties differentiate a PCS antenna from the existing smart antennas, and make it possible to implement a PCS antenna on a small-sized, low-power silicon chip.
\end{abstract}

\section{INTRODUCTION}

A wireless sensor network (WSN) is composed of several geographically distributed tiny sensors, where each sensor device is equipped with sensing, data processing and communication elements. WSNs have been extensively studied for many years due to their broad range of civil and military applications such as security surveillance, object detection, target tracking, home automation, environmental monitoring and health monitoring [1], [2], [3].

Conventional antennas for wireless transmission, e.g. omni-directional antennas, radiate in almost all directions. To avoid co-channel interference and unnecessary power consumption in undesired directions, a great deal of effort has been made in the past several decades to design smart transmitting/receiving antenna systems [4]. Two main types of smart antennas are switched beam and adaptive array. A switched beam smart antenna has several pre-designed fixed beam patterns, whereas an adaptive array smart antenna adaptively steers the beam to any direction of interest while simultaneously nulling interfering signals [5], [6]. An array system comprises multiple active (antenna) elements for varying the relative phases and amplitudes of the respective signals in order to generate a desired radiation pattern. Other types of smart antenna systems employ only one active element surrounded by a number of passive parasitic elements, with the disadvantage that their programming leads to an NP-hard problem [7], [8].

Javad Lavaei and John C. Doyle are with the Department of Control and Dynamical Systems, California Institute of Technology (emails: lavaei@cds.caltech.edu, doyle@cds.caltech.edu). Aydin Babakhani and Ali Hajimiri are with the Department of Electrical Engineering, California Institute of Technology (emails: aydin@caltech.edu, hajimiri@caltech.edu).
Different types of smart antennas, such as directional, beamforming/array, and multiple-input-multiple-output antennas, have been studied and applied to ad-hoc networks [9], [10]. The deployment of smart antennas is more crucial in WSNs than in general ad-hoc networks, due to very limited resources available for WSNs. A number of works have explored the synthesis of smart antennas in the context of WSNs. For instance, the papers [11], [12] study the necessity of changing the existing medium access control (MAC) protocols and the work [13] investigates the maximum flow problem under switched-beam directional antennas. Nonetheless, the smart antenna systems surveyed above cannot be exploited in WSNs by virtue of the fact that these systems either need heavy computations for their programming or are very large in size due to using several active elements (array elements) where the distance between neighboring elements is on the order of the signal wavelength.

In our previous works [14], [15], [16], we developed a rigorous method based on convex optimization to synthesize antenna systems efficiently. We built on these results in [17] and proposed a new type of smart antenna. This antenna system is referred to as passively controllable smart (PCS) antenna, which has a low-complex programming and utilizes only one active element. In [17], we demonstrated that PCS antennas have very powerful capabilities in spite of using a single active element, particularly in making a null at many (at least 10) directions. However, a PCS antenna is equipped with a programmable controller, which was assumed to be centralized in our previous work. The implementation of a centralized controller for an antenna system could be very costly and sometimes practically impossible. The objective of the present paper is to study how to design a PCS antenna with a decentralized (distributed) controller that is both practically implementable and easily programmable. This PCS antenna system can be implemented on a cheap, small-sized, low-power silicon chip to comply with the strict size and power limitations in WSNs.

\section{PCS ANTENNA SySTEMS}

A PCS antenna is a tiny system as small as one wavelength that is composed of the following components [17]:

- A dipole transmitting antenna: This dipole antenna is the only active element of the PCS antenna system, which is driven by a sinusoidal voltage source.

- A number of reflectors (or a patch array, alternatively): These reflectors surround the dipole antenna to shape the electromagnetic field in the space.

- A number of controllable ports (parasitic elements): These ports are mounted on the reflectors whose control 


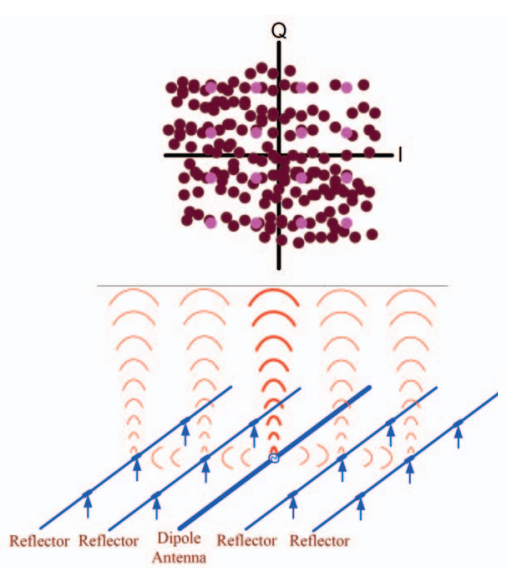

(a)

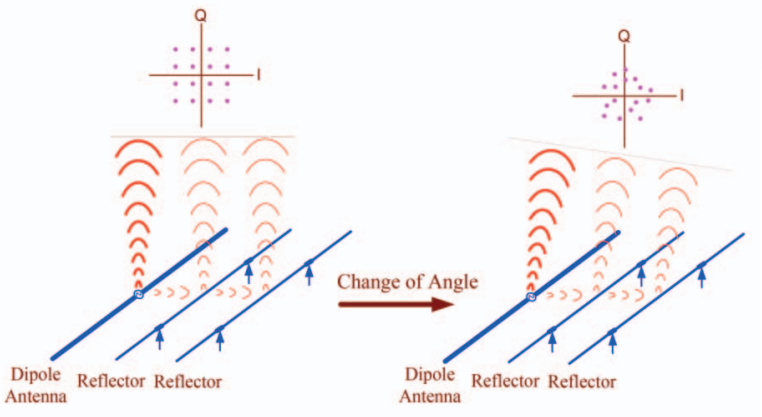

(b)

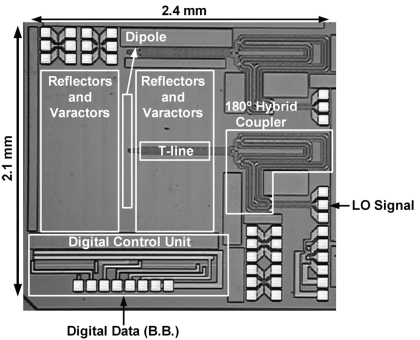

(c)

Fig. 1. (a): This figures illustrates the modulation capability of a PCS antenna system. (b): This figure illustrates the direction-dependent transmission capability of a PCS antenna. (c): This figure shows the micrograph of a PCS antenna system implemented in [8].

at every signal transmission could potentially form a desired radiation pattern at the far field.

- An adjustable passive network (controller): This passive network consists of passive elements, e.g. switches, resistors, capacitors and inductors, and is connected to the controllable ports of the reflectors to control the antenna system for every signal transmission.

Figure 1(a) exemplifies a PCS antenna system with 4 reflectors and 12 controllable ports (shown by arrows), where the passive controller consists of 12 ideal switches connected to the controllable ports. It can be observed that there exist $2^{12}$ switching combinations, each of which creates a specific point in the constellation digram. This modulation property of a PCS antenna has been throughly investigated in [18], where it is demonstrated that this type of antenna can also be used for direction-dependent data transmission. Figure 1(b) illustrates the latter property by showing that the constellation diagrams seen at different directions might be uncorrelated. The feasibility of implementing a PCS antenna on a silicon-based chip has been verified in [8], leading to the chip micrograph provided in Figure 1(c).

Consider a PCS antenna system with a pre-specified configuration (geometric shape). The act of changing the parameters in the passive controller of this PCS antenna system, which consequently modifies the radiation pattern generated at the far field, is referred to as the programming of the PCS antenna. Let $\mathcal{N}$ be a wireless network with $z+1$ nodes labeled as $0,1,2, \ldots, z$, where $z$ is a natural number. Assume that these nodes are geographically distributed so that none of the two nodes in the set $\{1,2, \ldots, z\}$ are co-linear with node 0 . This assumption is made to ensure an angle diversity among nodes $1,2, \ldots, z$ with respect to the reference node 0 . Equip node 0 with a transmitting PCS antenna and each of the remaining nodes with a simple dipole antenna for signal reception. In the rest of the paper, we study how the PCS antenna of node 0 can be programmed (online) so that it transmits data to an arbitrary node $j \in\{1,2, \ldots, z\}$ in such a

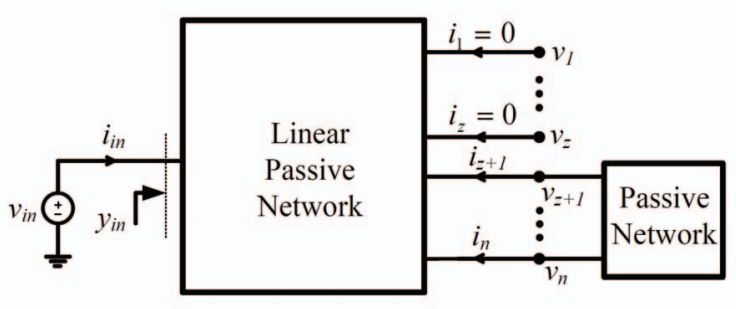

Fig. 2. Equivalent circuit model of a PCS antenna system.

way that many of the remaining nodes $1, \ldots, j-1, j+1, \ldots, z$ receive a zero signal.

\section{PRogramming OF PCS Antennas}

To study the programming capabilities of a PCS antenna system, the first step is to extract the equivalent circuit model of the entire antenna configuration at a desirable frequency $f_{0}$. This circuit model is given in Figure 2, where

- The block "Linear Passive Network" corresponds to the $Y$-parameter matrix of the antennas configuration.

- $v_{1}, \ldots, v_{z}$ denote the voltages induced on the receiving antennas of nodes $1,2, \ldots, z$, respectively.

- $v_{z+1}, v_{z+2}, \ldots, v_{n}$ denote the voltages on the controllable ports of the PCS antenna of node 0 (it is assumed that there are $n-z$ controllable ports).

- $v_{\text {in }}$ is the magnitude of the sinusoidal voltage driving the sole active element of the PCS antenna system.

- The block "Passive Network" represents the adjustable passive controller applied to the controllable ports of the PCS antenna of node 0 .

With no loss of generality, assume that $v_{\text {in }}=1$. Denote the $Y$-parameter matrix of the antennas configuration at the given frequency $f_{0}$ with $Y_{s}$.

As far as the implementation is concerned, the simplest type of the passive controller that one can envisage for a PCS antenna system is likely a switching controller, where each controllable port is connected to an ideal on/off switch. Nonetheless, it follows from [16] that designing a beamsteerable switching controller is indeed an NP-complete 
problem. This implies that the programming of the PCS antenna system using switching controllers is almost impossible in presence of even a moderate number of controllable ports (say 30 ports). On the other hand, our recent paper [17] substantiates that the programming of the PCS antenna system via a general strictly passive controller is computationally easy. Before proceeding with the main results of the current paper, let this important result be outlined first.

\section{A. Programming of PCS Antennas via Passive Controllers}

Decompose the complex-valued matrix $Y_{s}$ in a block form as

$$
Y_{s}=\left[\begin{array}{lll}
W_{11} & W_{12} & W_{13} \\
W_{21} & W_{22} & W_{23} \\
W_{31} & W_{32} & W_{33}
\end{array}\right]
$$

where $W_{11} \in \mathbf{C}^{z \times z}, W_{22} \in \mathbf{C}^{(n-z) \times(n-z)}$ and $W_{33} \in \mathbf{C}$ (note that $\mathbf{C}$ denotes the set of complex numbers). Let some matrices be introduced in the sequel:

$$
\begin{aligned}
& K_{1}:=W_{31} W_{11}^{-1} W_{12}-W_{32}, K_{2}:=W_{21} W_{11}^{-1}, \\
& K_{3}:=W_{31} W_{11}^{-1}, Q:=\left(\operatorname{Re}\left\{W_{22}-W_{21} W_{11}^{-1} W_{12}\right\}\right)^{-1}, \\
& \mathbf{o}:=-\left[\operatorname{Re}\left\{\frac{1}{2} K_{1} Q K_{2}+K_{3}\right\} \quad \operatorname{Im}\left\{\frac{1}{2} K_{1} Q K_{2}+K_{3}\right\}\right],
\end{aligned}
$$

where $\operatorname{Re}\{\cdot\}$ and $\operatorname{Im}\{\cdot\}$ are matrix operators returning the real and imaginary parts of their complex arguments. Since the matrix $Q$ introduced above is positive definite, one can define $Q^{\frac{1}{2}}$ as the unique symmetric positive definite matrix whose square is equal to $Q$. Assume that $K_{2}$ has full column rank over the field of complex numbers (see [17] for the case when this assumption is violated). Given $l \in \mathbf{N}$ and distinct indices $j, k \in\{1,2, \ldots, l\}$, define $\mathcal{P}_{j k}^{l}$ as a plane in $\mathbf{R}^{l}$ consisting of all vectors whose elements with the indices in the set $\{1,2, \ldots, l\} \backslash\{j, k\}$ are equal to zero. Moreover, let $\mathcal{D}$ be an ellipsoid encompassing all vectors $\mathbf{h} \in R^{1 \times 2 z}$ for which

$$
(\mathbf{h}-\mathbf{o})\left[\begin{array}{cc}
\operatorname{Re}\left\{K_{2}^{*} Q K_{2}\right\} & \operatorname{Im}\left\{K_{2}^{*} Q K_{2}\right\} \\
-\operatorname{Im}\left\{K_{2}^{*} Q K_{2}\right\} & \operatorname{Re}\left\{K_{2}^{*} Q K_{2}\right\}
\end{array}\right]^{-1}(\mathbf{h}-\mathbf{o})^{*}
$$

is less than $\frac{1}{4}\left\|K_{1} Q^{\frac{1}{2}}\right\|^{2}$ (where $\|\cdot\|$ represents the matrix 2-norm and the symbol "*" stands for the matrix conjugate transpose). We proved the following theorem in [17].

Theorem 1: A set of voltages $\left(v_{1}, v_{2}, \ldots, v_{z}\right)$ can be induced on the antennas of nodes $1,2, \ldots, z$ via a strictly passive control of the PCS antenna of node 0 if and only if the real-valued vector

$$
\left[\begin{array}{llllll}
\operatorname{Re}\left\{v_{1}\right\} & \cdots & \operatorname{Re}\left\{v_{z}\right\} & \operatorname{Im}\left\{v_{1}\right\} & \cdots & \operatorname{Im}\left\{v_{z}\right\}
\end{array}\right]
$$

belongs to the open ellipsoid $\mathcal{D}$.

This theorem characterizes the set of all voltages $\left(v_{1}, v_{2}, \ldots, v_{z}\right)$ that can be generated at the far field in different directions. For instance, the following properties can be inferred for every $j \in\{1,2, \ldots, z\}$ :

- The projection of the ellipsoid $\mathcal{D}$ on the plane $\mathcal{P}_{j(z+j)}^{2 z}$ leads to a circle, which is indeed the set of all possible complex voltages $v_{j}$ that can be generated by a strictly passive control of the PCS antenna.
- The intersection of the ellipsoid $\mathcal{D}$ with the plane $\mathcal{P}_{j(z+j)}^{2 z}$ (if nonempty) indeed corresponds to the set of all possible complex voltages $v_{j}$ that can be generated by a strictly passive control of the PCS antenna in such a way that other nodes $1, \ldots, j-1, j+1, . ., z$ receive a zero signal.

Although these properties indicate that the online programming of a PCS antenna system via a general passive controller is computationally easy (as complex as quadratic programming), the implementation of a general passive controller can be very difficult. In particular, a general passive controller may require mutual impedances between every two controllable ports, which cannot be implemented directly. In addition, such a controller needs as high as $\frac{(n-z)^{2}}{2}$ impedances, which could be a very large number.

\section{B. Programming of PCS Antennas via Decoupled Passive Controllers}

The above useful theoretical result will be exploited in this part to deign a practically implementable controller for a PCS antenna system. The type of the controller being sought is a decoupled (decentralized) passive controller, comprising $n-z$ (variable) impedances each for one of the controllable ports. As discussed in the introduction, the paper [8] has already demonstrated the feasibility of implementing such a PCS antenna system.

With no loss of generality, assume that the data transmission is supposed to occur between nodes 0,1 and the remaining nodes $2, \ldots, z$ act as unintended users. Given some nonnegative permissible tolerance $\varepsilon$, let $\mathcal{S}_{f}$ denote the set of all voltages $v_{1}$ that can be generated by the PCS antenna of node 0 under a decoupled passive controller subject to the constraint $\left\|v_{j}\right\| \leq \varepsilon$ for all $j \in\{2,3, \ldots, z\}$. The set $\mathcal{S}_{f}$ is indeed a constellation digram associated with $v_{1}$, which characterizes all possible signals that can be transmitted to node 1 by the PCS antenna in such a way that unwanted nodes $2, \ldots, z$ receive arbitrarily low-power signals. Consider the circuit in Figure 2 as well as the parameters $v_{2}, \ldots, v_{n}$ and $i_{z+1}, \ldots, i_{n}, i_{\text {in }}$ shown in this circuit. It can be verified that a complex-valued voltage $v_{1}$ belongs to $\mathcal{S}_{f}$ if and only if there exist voltages $v_{2}, \ldots, v_{n}$, currents $i_{z+1}, \ldots, i_{n}, i_{\text {in }}$ and a complex-valued diagonal matrix $D=\operatorname{diag}\left(d_{z+1}, \ldots, d_{n}\right)$ such that

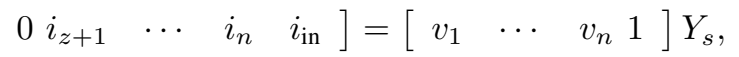

$$
\begin{aligned}
& {\left[\begin{array}{lll}
i_{z+1} & \cdots & i_{n}
\end{array}\right]=-\left[\begin{array}{lll}
v_{z+1} & \cdots & v_{n}
\end{array}\right] D,} \\
& \operatorname{Re}\left\{d_{j}\right\} \geq 0, \quad j=z+1, \ldots, n, \\
& \left\|v_{k}\right\| \leq \varepsilon, \quad k=2,3, \ldots, z
\end{aligned}
$$

where "0" in the equation (1a) represents a zero vector in $\mathbf{R}^{z}$. Note that the matrix $D$ in the above equations plays the role of the admittance matrix of the controller being applied to the PCS antenna $\left(d_{z+1}, \ldots, d_{n}\right.$ are the values of the admittances to be connected to the controllable ports). To simplify the above algebraic equations, it can be argued that a voltage $v_{1}$ belongs to $\mathcal{S}_{f}$ if and only if there exist voltages $v_{2}, \ldots, v_{n}$ 
and currents $i_{z+1}, \ldots, i_{n}, i_{\text {in }}$ such that

$$
\begin{aligned}
& {\left[\begin{array}{lllll}
0 & i_{z+1} & \cdots & i_{n} & i_{\text {in }}
\end{array}\right]=\left[\begin{array}{llll}
v_{1} & \cdots & v_{n} & 1
\end{array}\right] Y_{s}} \\
& \operatorname{Re}\left\{v_{j} i_{j}^{*}\right\} \leq 0, \quad j=z+1, \ldots, n, \\
& \left\|v_{k}\right\| \leq \varepsilon, \quad k=2,3, \ldots, z .
\end{aligned}
$$

Once the new set of equations in (2) is solved, the parameters $d_{1}, \ldots, d_{n-z}$ can be found using the relations $d_{j}=\frac{-v_{j}}{i_{j}}, j=$ $z+1, \ldots, n$. In order to study the true complexity of solving these equations, notice that:

- There are $4 n-2 z+2$ real variables involved in (2) as

$$
\begin{aligned}
& \operatorname{Re}\left\{v_{1}\right\}, \ldots, \operatorname{Re}\left\{v_{n}\right\}, \operatorname{Im}\left\{v_{1}\right\}, \ldots, \operatorname{Im}\left\{v_{n}\right\}, \operatorname{Re}\left\{i_{\text {in }}\right\}, \\
& \operatorname{Im}\left\{i_{\text {in }}\right\}, \operatorname{Re}\left\{i_{z+1}\right\}, \ldots, \operatorname{Re}\left\{i_{n}\right\}, \operatorname{Im}\left\{i_{n+1}\right\}, \ldots, \operatorname{Im}\left\{i_{n}\right\} .
\end{aligned}
$$

Let these variables be stacked in a vector, referred to as vector of variables.

- The inequalities (2b) or equivalently

$$
\operatorname{Re}\left\{v_{j}\right\} \operatorname{Re}\left\{i_{j}\right\}+\operatorname{Im}\left\{v_{j}\right\} \operatorname{Im}\left\{i_{j}\right\} \leq 0, \quad j=z+1, \ldots, n
$$

are the nonlinear constraints appearing in (2).

Define $\mathcal{C}_{1}$ as the set of all vectors of variables for which the constraints (2a) and (2c) hold. Likewise, let $\mathcal{C}_{2}$ be the set of those vectors of variables that satisfy the constraint (3). It can be seen that $\mathcal{S}_{f}$ is indeed the projection of the vector set $\mathcal{C}_{1} \cap \mathcal{C}_{2}$ on the plane $\mathcal{P}_{1(n+1)}^{4 n-2 z+2}$ (associated with $v_{1}$ ). However, although $\mathcal{C}_{1}$ is a convex set, the set $\mathcal{S}_{f}$ might be highly non-convex due to the non-convexity of the set $\mathcal{C}_{2}$. To investigate the shape of the region $\mathcal{C}_{2}$, assume for simplicity that $Y_{s}$ and $D$ are both real-valued, which make all variables in the system become real. Now, the feasibility region $\mathcal{C}_{2}$ corresponds to the constraints $v_{j} i_{j} \leq 0, \quad j=z+1, \ldots, n$, which are satisfied as long as $v_{j}$ and $i_{j}$ have opposite signs. Hence, $\mathcal{C}_{2}$ turns out to be the union of $2^{n-z}$ convex sets (orthants) glued together at the origin. This clearly shows the complexity of the shape of $\mathcal{C}_{2}$ in the general case. Hence, characterizing the exact shape of $\mathcal{S}_{f}$ could be formidable for a general admittance matrix $Y_{s}$.

Since $Y_{s}$ in this paper corresponds to a particular antenna configuration, this matrix has a nice hidden structure inherited from the associated Maxwell's equation. This could potentially help simplify the nonconvexity of the problem. Note that although the exact shape of $\mathcal{S}_{f}$ is unknown, we already know that this region is contained in the convex set $\mathcal{D} \cap \mathcal{P}_{1(z+1)}^{2 z}$ (because the set of passive controllers contains the set of decoupled passive controllers). In what follows, we propose a simple algorithm to find a subset of $\mathcal{S}_{f}$. It will be later verified in simulations that this algorithm often identifies a big part of $\mathcal{D} \cap \mathcal{P}_{1(z+1)}^{2 z}$ as a subset of $\mathcal{S}_{f}$, and hence the subset obtained must cover most of the set $\mathcal{S}_{f}$.

Algorithm 1:

Step 1: Generate a decoupled resistive network $D_{\text {init }}$ at random and apply the controller $D=D_{\text {init }}$ to the PCS antenna. Denote the signs of the real and imaginary parts of the obtained voltage $v_{j}$ with $t_{j}$ and $\bar{t}_{j}$, respectively, for every $j=z+1, \ldots, n$.

Step 2: Define $\alpha_{1}:=\operatorname{Re}\left\{v_{1}\right\}, \alpha_{2}:=\operatorname{Im}\left\{v_{1}\right\}, \alpha_{3}:=$ $-\operatorname{Re}\left\{v_{1}\right\}$ and $\alpha_{4}:=-\operatorname{Im}\left\{v_{1}\right\}$.
Step 3: For $i=1,2,3,4$, maximize $\alpha_{i}$ subject to the constraints $(2 \mathrm{a}),(2 \mathrm{c})$ and

$$
\begin{aligned}
& \operatorname{Re}\left\{v_{j}\right\} t_{j} \geq 0, \quad \operatorname{Im}\left\{v_{j}\right\} \bar{t}_{j} \geq 0, \\
& \operatorname{Re}\left\{i_{j}\right\} t_{j} \leq 0, \quad \operatorname{Im}\left\{i_{j}\right\} \bar{t}_{j} \leq 0, \quad j=z+1, \ldots, n .
\end{aligned}
$$

Denote the optimal value of $v_{1}$ with $q_{i}$

One can verify that Algorithm 1 amounts to one linear programming optimization (Step 1) and four secondorder-cone programming optimizations (Step 3). Hence, the complex numbers $q_{1}, q_{2}, q_{3}, q_{4}$ can all be found efficiently. Note that the constraint (4) in the optimizations defined in Step 3 is feasible because the voltages associated with the initial resistive controller $D_{\text {init }}$ form a feasible point for this constraint. From now on, identify the complex set $\mathcal{S}_{f}$ by a real region in $\mathbf{R}^{2}$.

Theorem 2: The polygon with the four vertices $\left(\operatorname{Re}\left\{q_{i}\right\}, \operatorname{Im}\left\{q_{i}\right\}\right), \quad i=1,2,3,4$, is contained in the constellation set $\mathcal{S}_{f}$.

Proof: Given $j \in\{z+1, \ldots, n\}$, the first observation is that the inequality (3) holds for some scalars $\operatorname{Re}\left\{v_{j}\right\}, \operatorname{Im}\left\{v_{j}\right\}, \operatorname{Re}\left\{i_{j}\right\}, \operatorname{Im}\left\{i_{j}\right\}$ if these quantities satisfy the constraint (4). Hence, the feasibility region $\mathcal{C}_{1} \cap \mathcal{C}_{2}$ contains the set of all vectors of variables for which the constraints specified in Step 3 of Algorithm 1 are satisfied. The next observation is that the new constraints all together form a convex feasibility region. The proof is completed by noting that Step 3 in Algorithm 1 intends to find four extreme points of this convex region.

It is noteworthy that designing an appropriate matrix $D$ corresponding to any specific point in the polygon given in Theorem 2 is equivalent to solving a second-order-cone optimization.

As spelled out earlier, $\mathcal{S}_{f}$ could be highly non-convex in light of the complicated shape of $\mathcal{C}_{2}$. On the other hand, since the binary vector $\left[\begin{array}{lllll}t_{z+1} & \cdots & t_{n} & \bar{t}_{z+1} & \cdots \bar{t}_{n}\end{array}\right]$ can take as high as $2^{2(n-z)}$ values, the set $\mathcal{C}_{2}$ might be composed of an exponential number of convex regions glued together. Due to the fact that Algorithm 1 considers merely one possible value for this vector, it may be speculated that this algorithm can find only a small portion of $\mathcal{S}_{f}$; however, it will be verified through simulations that the algorithm can find a big part of $\mathcal{S}_{f}$. Indeed, although $\mathcal{S}_{f}$ is not known exactly, we use Theorem 1 to find a limit for $\mathcal{S}_{f}$ and then compare it with the polygon obtained in Theorem 2 .

\section{Simulation Results}

Two examples will be presented in this section to study the modulation (Example 1) and beam steering (Example 2) capabilities of a PCS antenna under a decentralized controller.

Example 1: Consider the PCS antenna system depicted in Figure 3(a), which consists of a transmitting dipole antenna and 10 metal reflectors each with 5 ports (antenna parasitic elements). The objective is to transmit data from this PCS antenna to a receiving dipole antenna located at the far field in the upward direction. Assume that the PCS antenna system is driven by a sinusoidal voltage source with the frequency $60 \mathrm{GHz}$ and the amplitude of 1 volt. The circuit model of 


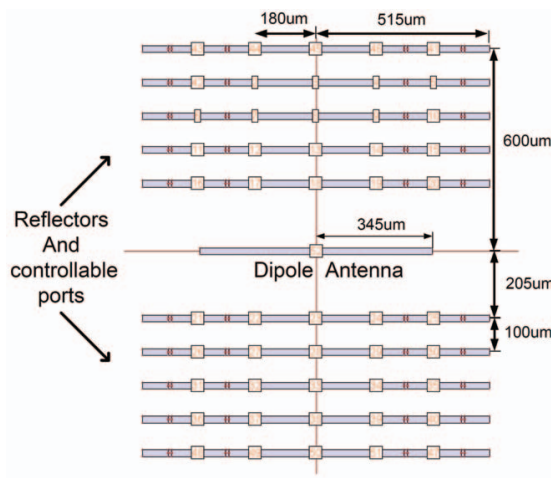

(a)

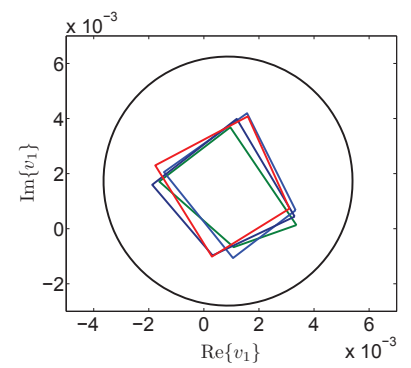

(b)

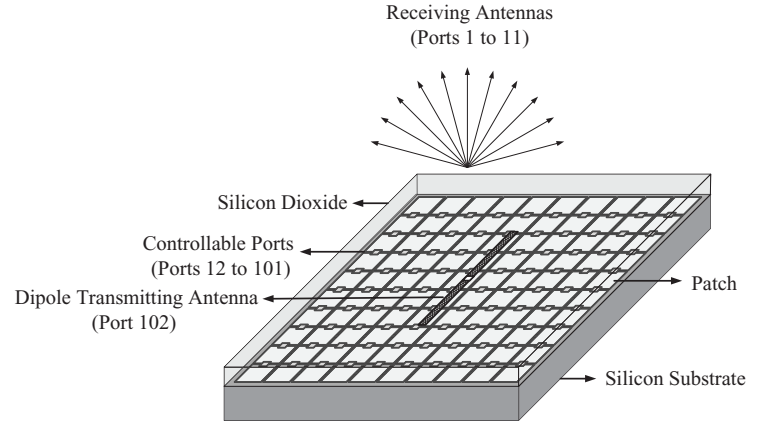

(c)

Fig. 3. (a): The antenna system studied in Example 1; (b): The constellation diagram for $v_{1}$ (Example 1); (c): The PCS antenna studied in Example 2.

the antenna system can be extracted at the desired frequency $60 \mathrm{GHz}$ by means of the electromagnetic software IE3D [19]. Using Theorem 1, the set of all possible voltages $v_{1}$ that can be generated by the PCS antenna under a general passive controller is obtained as the circle depicted in Figure 1(b).

Since a general passive controller may not be implementable in practice (partially due to requiring about $50 \times 25$ impedances), the goal is to find a convex subset of $\mathcal{S}_{f}$, where $\mathcal{S}_{f}$ is defined as the set of those voltages $v_{1}$ that can be generated via a decoupled controller with 50 impedances. First, recall that $\mathcal{S}_{f}$ must be contained in the circle obtained using Theorem 1. Algorithm 1 is run four times and the corresponding polygons are drawn in Figure 3(b). It can be observed that all these polygons are very close to each other and that these polygons cover a big part of the circle. Any voltage point inside these polygons can be generated by a diagonal admittance matrix $D$ found by solving a linear programming optimization. Note that each of these polygons is indeed a feasible constellation diagram for $v_{1}$, which can be used to find a signal with the maximum received power or to attain the maximum number of symbols that can be modulated/demodulated reliably. A similar analysis can be performed for the input admittance of the antenna, which is useful for the antenna impedance matching problem.

Example 2: Consider a cluster of 12 wireless nodes, labeled as $0,1, \ldots, 11$, which are distributed in a plane with the configuration given in Figure 4(a). We equip nodes $1,2, \ldots, 11$ with simple dipole antennas for signal reception and node 0 with a PCS antenna for signal transmission. This PCS antenna has 90 controllable ports and is depicted in Figure 3(c) (see [15] for the detailed specifications of the transmitting and receiving antennas used in this example). The goal is to transmit data from node 0 to node 6 in such a way that some of the remaining nodes receive a zero signal, if possible. To this end, Theorem 1 yields that the set of all voltages $v_{6}$ that can be generated using a passive control of the PCS antenna is the circle given in Figure 4(b). Every run of Algorithm 1 identifies a subset this circle that can be generated using a decoupled passive controller with 90 impedances (rather than about $90 \times 45$ impedances). We run the algorithm twice and obtained the two polygons sketched in Figure 4(b). The union of these polygons is a feasible constellation digram for $v_{6}$ corresponding to the decoupled passive control of the PCS antenna.

As the second objective, we wish to find a set of the voltages $v_{6}$ that can be generated via the PCS antenna of node 0 in such a way that a node $k \in\{1,2, \ldots, 5,7, \ldots, 11\}$ receives a zero signal. We run Algorithm 1 twice corresponding to each node $k$ and observed that as long as $k$ is different from 3 and 7 , this goal is achievable. The corresponding polygons obtained for $k=2,4,5,8$ are depicted in Figures 4(c) and 5(a)-(c). In each of these figures, the two colored (inner) polygons show the polygons obtained using Algorithm 1 and the black (outer) polygon represents the union of the polygons obtained in Figure 4(b) corresponding to the case when there is no constraint on the voltage of node $k$. It is even possible to make a null at multiple directions simultaneously. For instance, the constellation digram for $v_{6}$ subject to the null constraints $v_{2}=v_{8}=0$ is depicted in Figure 5(d). Node 0 can compute this diagram online to program its PCS antenna to send data to node 6 in such a way that nodes 2 and 8 both receive no signal. Although Algorithm 1 was run twice in each of the foregoing cases, we observed through extensive simulations that more runs of the algorithm do not enlarge the constellation diagram noticeably.

\section{CONCLUSIONS}

We recently introduced a secure, power-efficient, beamsteerable and on-chip antenna, referred to as passively controllable smart (PCS) antenna system. A PCS antenna system can be programmed to generate different radiation patterns at the far field by adjusting its variable passive controller at every signal transmission. In particular, the PCS antenna is able to transmit data to a desired direction in such a way that no signal is sent in pre-specified undesired directions. We allowed the controller of a PCS antenna to be centralized in our previous work, which was an obstacle to the implementation of this controller. In this work, the design of PCS antenna systems under decentralized controllers is studied with the aim of facilitating the implementation and reducing the number of electrical elements used in the controller. Unlike 


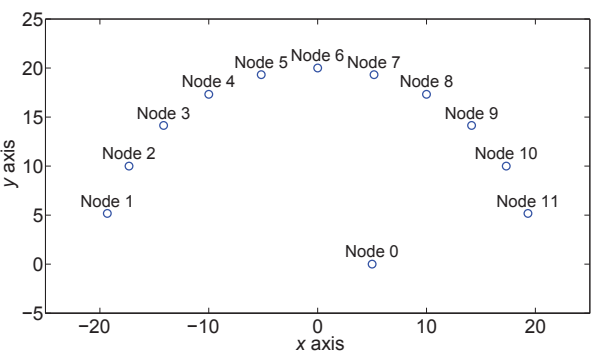

(a)

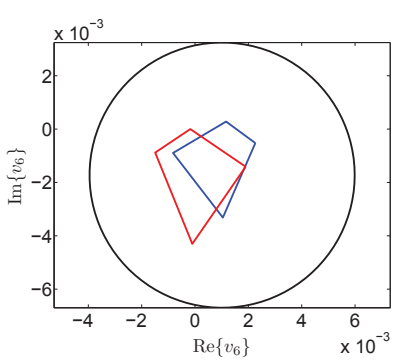

(b)

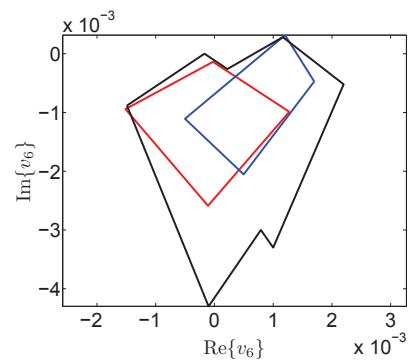

(c)

Fig. 4. (a): The configuration of the transmitting and receiving nodes in Example 2; (b): The constellation diagram for $v_{6}$ (Example 2); (c): The constellation diagram for $v_{6}$ under the constraint $v_{2}=0$ (Example 2).

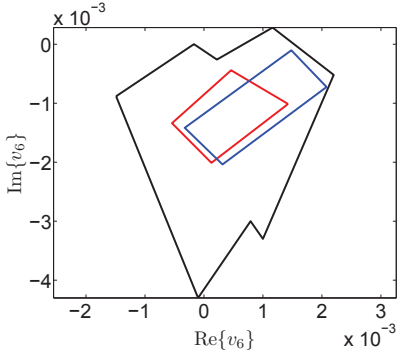

(a)

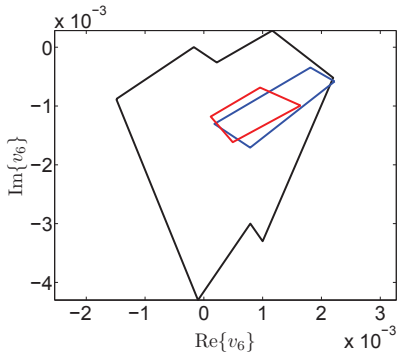

(b)

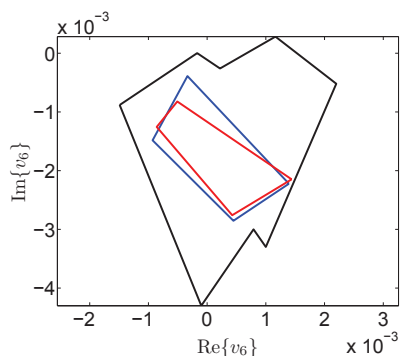

(c)

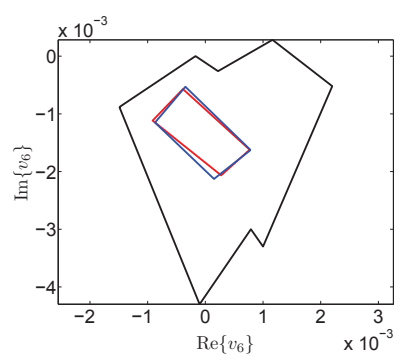

(d)

Fig. 5. (a): The constellation diagram for $v_{6}$ under the constraint $v_{4}=0$; (b): The constellation diagram for $v_{6}$ under the constraint $v_{5}=0$; (c): The constellation diagram for $v_{6}$ under the constraint $v_{8}=0$; (d): The constellation diagram for $v_{6}$ under the constraints $v_{2}=0$ and $v_{8}=0$ (Example 2).

the existing smart antennas whose programming leads to an NP-hard problem or are made of many active elements, the PCS antenna proposed here has a low-complex programming capability and consists of only one active element.

\section{ACKNOWLEDGMENTS}

This research was supported by ONR MURI N00014-081-0747 "Scalable, Data-driven, and Provably-correct Analysis of Networks," ARO MURI W911NF-08-1-0233 “Tools for the Analysis and Design of Complex Multi-Scale Networks," and the Army's W911NF-09-D-0001 Institute for Collaborative Biotechnology.

\section{REFERENCES}

[1] E. H. Callaway, "Wireless sensor networks: architectures and protocols," CRC Press, 2003.

[2] I. Stojmenovic, "Handbook of sensor networks: algorithms and architectures," John Wiley \& Sons, 2005.

[3] I. F. Akyildiz, W. Su, Y. Sankarasubramaniam and E. Cayirci, "A survey on sensor networks," IEEE Communications Magazine, vol. 40, no. 8, pp. 102-114, 2002.

[4] J. C. Liberti and T. S. Rappaport, "Smart antennas for wireless communications: IS-95 and third generation CDMA applications," Prentice Hall, 1999.

[5] L. C. Godara, "Smart antennas," CRC Press, Boca Raton, 2004.

[6] A. E. Zooghby, "Smart antenna engineering," Artech House: Norwood, 2005.

[7] T. Ohira and K. Gyoda, "Electronically steerable passive array radiator antennas for low-cost analog adaptive beamforming," IEEE International Conference on Phased Array Systems and Technology, Dana Point, CA, 2000.

[8] A. Babakhani, D. B. Rutledge and A. Hajimiri, "Near-field direct antenna modulation, IEEE Microwave Magazine, vol. 10, no. 1, pp. 36-46, 2009.
[9] K. Sundaresan and R. Sivakumar, "A unified MAC layer framework for ad-hoc networks with smart antennas," IEEE/ACM Transactions on Networking, vol. 15, no. 3, pp. 546-559, 2007.

[10] J. Iguchi-Cartigny, P. M. Ruiz, D. Simplot-Ryl, I. Stojmenovic and C. M. Yago, "Localized minimum-energy broadcasting for wireless multihop networks with directional antennas," IEEE Transactions on Computers, vol. 58, no. 1, pp. 120-131, 2009.

[11] G. Boudour, A. Lecointre, P. Berthou, D. Dragomirecu and T. Gayraud, "On designing sensor networks with smart antennas," IFAC International Conference on Fieldbuses and Networks in Industrial and Embedded Systems, Toulouse, France, 2007.

[12] C. Santivanez and J. Redi, "On the use of directional antennas for sensor networks," IEEE Military Communications Conference, Boston, MA, 2003.

[13] X. Huang, J. Wang and Y. Fang, "Achieving maximum flow in interference-aware wireless sensor networks with smart antennas," Ad Hoc Networks, vol. 5, no. 6, pp. 885-896, 2007.

[14] J. Lavaei, A. Babakhani, A. Hajimiri, and J. C. Doyle, "A study of near-field direct antenna modulation systems using convex optimization," American Control Conference, Baltimore, 2010.

[15] A. Babakhani, J. Lavaei, J. C. Doyle, and A. Hajimiri, "Finding globally optimum solutions in antenna optimization problems," IEEE International Symposium on Antennas and Propagation, Toronto, Canada, 2010.

[16] J. Lavaei, A. Babakhani, A. Hajimiri, and J. C. Doyle, "Solving largescale hybrid circuit-antenna problems," to appear in IEEE Transactions on Circuits and Systems I, 2010.

[17] J. Lavaei, A. Babakhani, A. Hajimiri, and J. C. Doyle, "Programmable antenna design using convex optimization," 19th International Symposium on Mathematical Theory of Networks and Systems, Budapest, Hungary, 2010.

[18] A. Babakhani, D. B. Rutledge and A. Hajimiri, "Transmitter architectures based on near-field direct antenna modulation," IEEE Journal of Solid-State Circuits, vol. 43, no. 12, pp. 2674-2692, 2008.

[19] IE3D electromagnetic simulation and optimization software, Zeland Software Inc., www.zeland.com. 